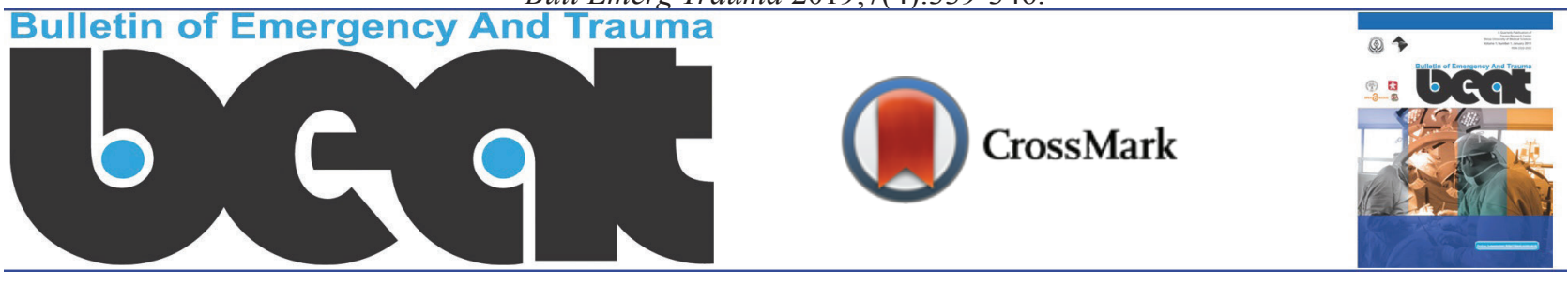

\title{
Drug Related Hospital Admissions; A Systematic Review of the Recent Literatures
}

\author{
Mohammed Biset Ayalew ${ }^{1}$, Henok Getachew Tegegn ${ }^{1}$, Ousman Abubeker Abdela ${ }^{1 *}$
}

${ }^{1}$ Department of Clinical Pharmacy, School of Pharmacy, College of Medicine and Health Sciences, Gondar University, Gondar, Ethiopia

*Corresponding author: Ousman Abubeker Abdela

Address: Department of Clinical Pharmacy, School of Pharmacy, College of

Medicine and Health Sciences, Gondar University, Gondar, Ethiopia. PO. Box 196.

Tel: +251-912-02-3692, Fax: 251-058-114 1240, e-mail: ousmy2009@gmail.com
Received: March 30, 2019

Revised: June 29, 2019

Accepted: July 10, 2019

\section{ABSTRACT}

Objective: To derive findings from different studies done on drug related hospital admissions and comprehensively express the incidence and preventability of drug related hospital admissions; identify the common types of drug related problems that caused hospital admission, and identify factors associated with drug related hospital admission.

Methods: Literatures that assessed hospitalization due to drug related problems were searched online using Pub Med and Google Scholar databases. The relevant reference lists of retrieved articles were also searched manually on Google. Prospective and retrospective studies conducted anywhere in the world on drug related hospitalization, published from January 2012 to January 2017 as an original article and written in English language were included.

Result: The prevalence of drug related hospital admission varies from $1.3 \%$ to $41.3 \%$ with the average rate of $15.4 \%$. Among hospitalized patients $2.7 \%$ were died due to drug-related problems (DRPs). Drugs that were frequently reported as causing drug related admission were antithrombotic drugs, antihypertensive drugs, analgesics, anti-diabetics, antipsychotics, and anti-neoplastic drugs. Poly pharmacy, old age and female sex were mentioned as determinants for drug related hospitalization by a number of studies. About one third of drug related hospital admissions were definitely preventable and more than $40 \%$ were also potentially preventable. Conclusion: Drug related problems contribute for more than $15 \%$ of hospital admissions. Higher risk of admission due to DRPs was observed in patients who were on poly pharmacy and those who were old. As most of drug related hospital admissions were preventable an emphasis should be given for preventive strategies to avoid complications and costs associated with admission.

Keywords: Drug related problems; Hospital admission; Adverse drug reaction; Review; Emergency visit.

Please cite this paper as:

Ayalew MB, Tegegn HG, Abdela OA. Drug Related Hospital Admissions; A Systematic Review of the Recent Literatures. Bull Emerg Trauma. 2019;7(4):339-346. doi: 10.29252/beat-070401.

Introduction

$\mathrm{W}$ orld-wide, medication use is increasing. This can be explained as a result of production of more types of medications by the advancing pharmaceutical industries and the ever increasing types of diseases that amplified needs of pharmaceuticals. Possible outcomes of medication use may range from the intended beneficial effect interventions to minor side effects and even death. 
Drug related problem (DRP) is defined as an event or circumstance that involves a patient's drug treatment that actually, or potentially, interferes with the achievement of an optimal outcome $[1,2]$. This can be inappropriate drug selection, adverse drug reactions, untreated indication, drug interactions, inappropriate dosage, drug use without indication and non-compliance.

Admissions due to DRPs have been reported as growing over the past decades [3]. In United States, estimates suggested that Drug-related problems (DRPs) accounted for 17 million emergency department visits and 8.7 million hospital admissions annually [4]. Evaluation of studies on DRPs which leads to hospital admission have indicated that DRPs are responsible for approximately $5-15 \%$ of all hospitalizations, of which $25-75 \%$ were avoidable [5-11]. Those medications with narrow therapeutic index and medications which require continuous and regular monitoring are the one most implicated in avoidable adverse drug events (ADEs) [5]. A number of factors have been implicated to be risk factors for drug related hospital admission. Examples include being old $[3,7,12,13]$, Poly pharmacy $[3,14]$, poly physician [14], being female [15], and presence of co morbidities $[5,16,17]$. Drug related hospitalization will have negative consequences on patients and society. It increases mortality and morbidity rates, health care cost, decreases income and household productivity and reduced quality of life $[5,18]$.

Different studies across the world reported varying level of incidence and preventability of drug related hospital admission based on the settings, methods used and populations addressed. It is very important to see the current overall picture of drug related hospitalization to understand the nature and extent of the problem and to devise strategies for preventing its harm. So the aim of this review is to derive findings from different studies done on drug related hospital admissions and comprehensively expresses the incidence and preventability of drug related hospital admissions; identify the common types of DRPs that caused hospital admission, and identify factors responsible for drug related hospital admission.

\section{Materials and Methods}

\section{Search Strategy}

Literatures that assessed admission to an emergency department or other units of the hospital due to adverse drug reactions or any other drug related problems were searched online using Pub-med and Google Scholar databases. The following words were used in different combinations during online search; Drug related problem(s), hospitalization, emergency department visit, adverse drug reaction, hospital admission and drug related admission. The relevant reference lists of retrieved articles were also searched manually on Google.

\section{Article Selection}

All of the identified articles were independently reviewed by three authors to assess eligibility for inclusion in the review. Disagreements were resolved by consensus. Prospective and retrospective studies conducted anywherein the world on drug related hospitalization, published in January 2012 to January 2017 as an original article and written in English language were included in the study. Studies conducted in the pediatric population were excluded.

\section{Data Extraction}

Information regarding study characteristics (study area, study design, subjects and sample size) and main findings (frequency of hospital admission and death due to drug related problems, type, severity, casualty and preventability of DRPs, drugs and drug classes responsible for admission and factors associated with drug related admission) were extracted from each studies.

\section{Results}

\section{Literature Search Results}

A total of 326 articles were obtained from Pub med, Google Scholar and manual Google search. After adjusting for duplicates 263 articles remained. Of these 214 studies were found irrelevant after reviewing their titles. Thirty-one studies were discarded as they do not satisfy the inclusion criteria after reviewing their abstracts. The full text of the remaining 18 studies was reviewed in detail. Two studies were removed after the full text was reviewed since it did not address many of the needed information. Finally, as shown in Figure 1, sixteen studies were found convenient to be included in this review.

\section{Study Characteristics}

Among the 16 studies reviewed majority (12) assessed admission to any department of the hospital

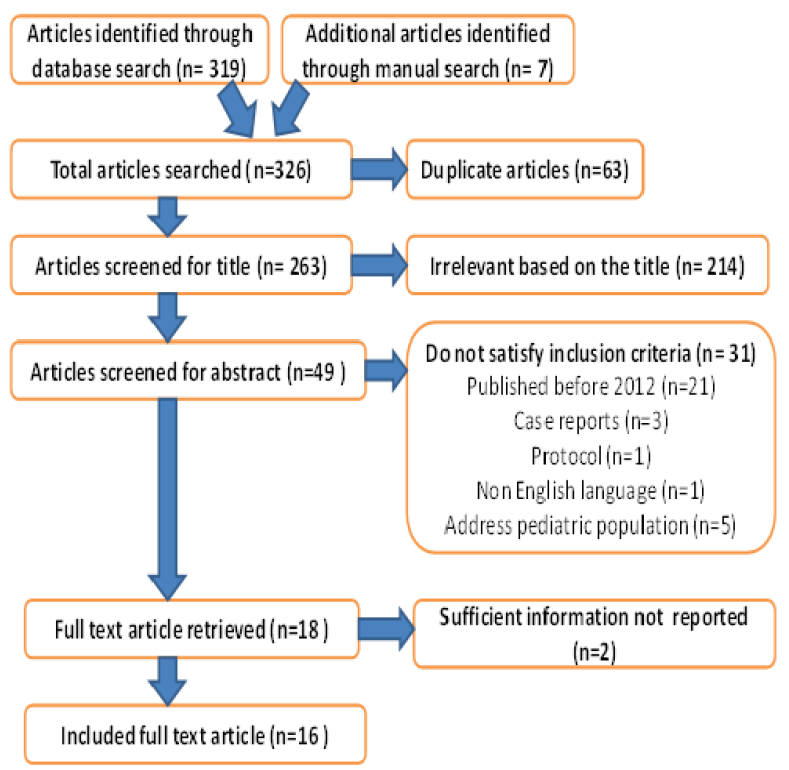

Fig. 1 Flow diagram of study selection. 
while 4 assessed admissions to emergency department only. Nine of the studies reported hospitalization due to any type of drug related problems and the other 7 assessed admissions due to adverse drug reactions only. Nine of the studies included all adult patients while others include only specific patient groups like cancer patients (1), cardiac transplanted patients (1), elderly patients with dementia (1), patient who are age 65 or more (2), age 60 or more (1) and age 55 or more (1). As shown in Table 1, the studies were conducted in different parts of the world on samples of $48-2,127,133$ patients. Most (11) of the studies were prospective in design.

\section{Drug Related Hospitalization and Death}

There is no significant difference in the rate of drug related hospitalization between the large and small sample size studies $(p=0.268)$. As indicated in Table 2 , the rate of drug related hospital admission varies from $1.3 \%$ in Netherlands to $41.3 \%$ in Sweden. The average rate of drug related hospital admission is $15.4 \%$. Seven studies reported that $0 \%$ up to $5.7 \%$ of patients hospitalized due to drug related problems were died. The average death rate due to DRPs in hospitalized patients is $2.7 \%$.

Drug Related Problems that Cause Hospital Admission

As shown in Table 3 the common drug related problems that caused hospital admission were adverse drug reaction, dosing problems, noncompliance, untreated indication and improper drug selection.

Table 1. Individual study characteristics

\begin{tabular}{|c|c|c|c|c|c|}
\hline $\begin{array}{l}\text { Sr. } \\
\text { no. }\end{array}$ & $\begin{array}{l}\text { Author, year of } \\
\text { publication }\end{array}$ & Study area/country & Study subjects & Study design & $\begin{array}{l}\text { Sample } \\
\text { size }\end{array}$ \\
\hline 1 & $\begin{array}{l}\text { Alghamdy M. et } \\
\text { al, } 2015[20]\end{array}$ & Saudi Arabia & $\begin{array}{l}\text { Admitted patients at emergency } \\
\text { department }\end{array}$ & Retrospective record review & 5622 \\
\hline 2 & $\begin{array}{l}\text { Chan A. et al, } 2014 \\
\text { [21] }\end{array}$ & $\begin{array}{l}\text { Singapore General } \\
\text { Hospital }\end{array}$ & $\begin{array}{l}\text { cancer patients admitted to two } \\
\text { oncology wards }\end{array}$ & $\begin{array}{l}\text { prospective, observational } \\
\text { study }\end{array}$ & 1274 \\
\hline 3 & $\begin{array}{l}\text { Schmiedl, S et al, } \\
2014 \text { [22] }\end{array}$ & German & $\begin{array}{l}\text { patients admitted to the internal } \\
\text { medicine departments }\end{array}$ & $\begin{array}{l}\text { multi-centre, Prospective } \\
\text { study }\end{array}$ & 212,000 \\
\hline 4 & $\begin{array}{l}\text { Benard-laribiere A. } \\
\text { etal, } 2015 \text { [23] }\end{array}$ & $\begin{array}{l}61 \text { Medical wards in } \\
\text { public hospitals of } \\
\text { France }\end{array}$ & $\begin{array}{l}\text { Patients admitted to the medical } \\
\text { wards }\end{array}$ & Prospective study & 2692 \\
\hline 5 & $\begin{array}{l}\text { ReppK.. et al, } \\
2012[5]\end{array}$ & $\begin{array}{l}\text { Saint Luke's North } \\
\text { Hospital, Kansas City, } \\
\text { USA }\end{array}$ & cardiac transplant patients & $\begin{array}{l}\text { prospective longitudinal - } \\
\text { single center study }\end{array}$ & 48 \\
\hline 6 & $\begin{array}{l}\text { Al-Arifi M. et al, } \\
2014 \text { [4] }\end{array}$ & Saudi Arabia & $\begin{array}{l}\text { Patients visiting emergency } \\
\text { department }\end{array}$ & $\begin{array}{l}\text { prospective cohort } \\
\text { observational study }\end{array}$ & 300 \\
\hline 7 & $\begin{array}{l}\text { Nickel C. et al, } \\
2013[26]\end{array}$ & $\begin{array}{l}\text { University Hospital } \\
\text { Basel , Switzerland }\end{array}$ & $\begin{array}{l}\text { non-trauma patients presenting } \\
\text { to the ED with non-specific } \\
\text { complaints }\end{array}$ & $\begin{array}{l}\text { Prospective crossectional } \\
\text { study }\end{array}$ & 633 \\
\hline 8 & $\begin{array}{l}\text { Gustafsson M. et } \\
\text { al, 2016[16] }\end{array}$ & Sweden & $\begin{array}{l}\text { Elderly patients with dementia or } \\
\text { cognitive impairment admitted } \\
\text { orthopedic and internal medicine } \\
\text { wards }\end{array}$ & NR & 458 \\
\hline 9 & $\begin{array}{l}\text { Jatau A. et al, } 2015 \\
{[18]}\end{array}$ & $\begin{array}{l}\text { UniversitiSains } \\
\text { Hospital, Malaysia }\end{array}$ & patients who visited the ED & $\begin{array}{l}\text { Prospective cross-sectional } \\
\text { study }\end{array}$ & 434 \\
\hline 10 & $\begin{array}{l}\text { Ruiter Ret al, } 2012 \\
{[10]}\end{array}$ & $\begin{array}{l}\text { Hospitals in } \\
\text { Netherlands }\end{array}$ & $\begin{array}{l}\text { patients } \geq 55 \text { years of age with an } \\
\text { acute, non-planned admission to a } \\
\text { Dutch hospital }\end{array}$ & Retrospective study & $\begin{array}{l}2, \\
127,133\end{array}$ \\
\hline 11 & $\begin{array}{l}\text { Pedrós C.et al, } \\
2016 \text { [24] }\end{array}$ & $\begin{array}{l}\text { Bellvitge University } \\
\text { hospital, Barcelona, } \\
\text { Spain. }\end{array}$ & $\begin{array}{l}\text { Patients aged } \geq 65 \text { years who were } \\
\text { urgently admitted at the hospital }\end{array}$ & $\begin{array}{l}\text { prospective cross-sectional } \\
\text { study }\end{array}$ & 60,263 \\
\hline 12 & $\begin{array}{l}\text { Ahern F, et al, } \\
2013[32]\end{array}$ & $\begin{array}{l}\text { Cork University } \\
\text { Hospital (CUH) } \\
\text { emergency department, } \\
\text { Ireland }\end{array}$ & Patients admitted to the ED & retrospective study & 856 \\
\hline 13 & $\begin{array}{l}\text { Pedrós C. et al, } \\
2014 \text { [25] }\end{array}$ & $\begin{array}{l}\text { Bellvitge University } \\
\text { Hospital, Spain }\end{array}$ & $\begin{array}{l}\text { patients admitted through the } \\
\text { emergency room }\end{array}$ & $\begin{array}{l}\text { prospective cross-sectional } \\
\text { study }\end{array}$ & 4,403 \\
\hline 14 & $\begin{array}{l}\text { Karuppannan M. et } \\
\text { al, } 2013 \text { [27] }\end{array}$ & $\begin{array}{l}\text { Two medical wards at a } \\
\text { government hospital in } \\
\text { Malaysia. }\end{array}$ & Patients admitted to medical wards & Prospective study & 1200 \\
\hline 15 & $\begin{array}{l}\text { Skoldunger A. et } \\
\text { al, } 2015 \text { [34] }\end{array}$ & four sites in Sweden & individuals aged $\geq 60$ years & $\begin{array}{l}\text { Longitudinal prospective } \\
\text { cohort study }\end{array}$ & 4108 \\
\hline 16 & $\begin{array}{l}\text { Marcum Z. et al, } \\
2012 \text { [33] }\end{array}$ & $\begin{array}{l}\text { Veterans Affairs (VA) } \\
\text { Medical Centers, USA }\end{array}$ & older (aged $\geq 65$ ) & Retrospective cohort & 678 \\
\hline
\end{tabular}

aUSA; United States of America 
Table 2. Rate of drug related hospital admission and death and types of DRPs causing hospitalization

\begin{tabular}{|c|c|c|c|c|}
\hline $\begin{array}{l}\text { Sr. } \\
\text { no. }\end{array}$ & $\begin{array}{l}\text { Author, year of } \\
\text { publication }\end{array}$ & $\begin{array}{l}\text { Admission to } \\
\text { Hospital (reason) }\end{array}$ & $\begin{array}{l}\text { Death due to } \\
\text { DRPs }\end{array}$ & Types of DRPs that causes admission \\
\hline 1 & $\begin{array}{l}\text { Alghamdy M. et al, } 2015 \\
\text { [20] }\end{array}$ & $\begin{array}{l}253(4.5 \%) \\
\text { (due to DRP) }\end{array}$ & $10(4 \%)$ & $\begin{array}{l}\text { Noncompliance } 112(44.3 \%) \\
\text { Toxicity and SEs } 50(19.8 \%) \\
\text { Drug-drug interactions } 29(11.5 \%) \\
\text { Accidental and suicidal drug ingestions } 26(10.3 \%) \\
\text { Drug abuse } 18(7.1 \%) \\
\text { Drug allergy } 10(4 \%) \\
\text { Super-infections } 8(3.2 \%)\end{array}$ \\
\hline 2 & Chan A. et al, 2014 [21] & $\begin{array}{l}158(12.4 \%) \\
\text { (due to DRP) }\end{array}$ & $5(3 \%)$ & $\begin{array}{l}\text { Adverse reaction } 155(94.5 \%) \\
\text { Drug interactions } 3(1.8 \%) \\
\text { Dosing problem } 3(1.8 \%) \\
\text { Drug use problem } 3(1.8 \%)\end{array}$ \\
\hline 3 & $\begin{array}{l}\text { Schmiedl, S et al, } 2014 \\
\text { [22] }\end{array}$ & $\begin{array}{l}\text { 6887(3.2\%) } \\
\text { (due to ADR) }\end{array}$ & $1.32 \%$ & NR \\
\hline 4 & $\begin{array}{l}\text { Benard-laribiere A. et al, } \\
2015 \text { [23] }\end{array}$ & $\begin{array}{l}97(3.6 \%) \\
\text { (due to } \mathrm{ADR})\end{array}$ & $1 \%$ & $\begin{array}{l}\text { Type A reactions } 67(69.1 \%) \\
\text { Vascular disorders } 20(20.6 \%) \\
\text { CNS disorders } 11(11.3 \%) \\
\text { Gastrointestinal disorders } 9(9.3 \%) \\
\text { General disorders } 9(9.3 \%) .\end{array}$ \\
\hline 5 & ReppK. et al, 2012 [5] & $\begin{array}{l}40 \%(19 / 48) \\
\text { (due to DRP) }\end{array}$ & $0 \%$ & $\begin{array}{l}\text { Adverse drug reactions } 6(32 \%) \text { Supra-therapeutic } \\
\text { doses } 6(32 \%) \\
\text { Sub-therapeutic doses } 3(16 \%) \text { Untreated indication } \\
1(5 \%) \\
\text { Non-adherence } 2(11 \%) \\
\text { Drug interactions } 1(5 \%)\end{array}$ \\
\hline 6 & Al-Arifi M. et al, 2014 [4] & $\begin{array}{l}56(18.7 \%) \\
\text { (due to DRPs) }\end{array}$ & NR & $\begin{array}{l}\text { Adverse drug reactions }(30.4 \%) \\
\text { non-compliance }(30.4 \%) \\
\text { Untreated indication }(10.7 \%) \\
\text { Drug interactions }(7.1 \%) \\
\text { Supra-therapeutic }(7.1 \%) \\
\text { Sub-therapeutic dose }(7.1 \%) \\
\text { Improper drug selection }(5.4 \%) \\
\text { drug use without indication }(1.8 \%)\end{array}$ \\
\hline 7 & Nickel C. et al, 2013 [26] & $\begin{array}{l}77(12.2 \%) \\
\text { (due to DRPs) }\end{array}$ & NR & $\begin{array}{l}\text { Adverse drug reactions } 42(56 \%) \\
\text { Drug choice problem } 9(12 \%) \\
\text { Dosing problems } 16(21 \%) \\
\text { Drug use problems } 2(3 \%) \\
\text { Drug interactions } 3(4 \%)\end{array}$ \\
\hline 8 & $\begin{array}{l}\text { Gustafsson M. et al, } 2016 \\
{[16]}\end{array}$ & $\begin{array}{l}189(41.3 \%) \text { (due to } \\
\text { DRPs) }\end{array}$ & NR & $\begin{array}{l}\text { Dosage too high }(12.7 \%) \text { Noncompliance }(10.6 \%) \\
\text { Ineffective drug } 10.6 \% \\
\text { interactions } 6.9 \% \\
\text { Needs additional drug }(6.3 \%) \\
\text { Dosage too low }(4.8 \%) \\
\text { Unnecessary drug therapy }(2.6 \%)\end{array}$ \\
\hline 9 & Jatau A. et al, 2015 [18] & $\begin{array}{l}133(30.6 \%) \\
\text { (due to ADE) }\end{array}$ & NR & $\begin{array}{l}\text { Therapeutic failure } 74(55.6 \%) \\
\text { ADR } 43(32.3 \%) \\
\text { Accidental overdose } 7(5.2 \%) \\
\text { Intentional overdose } 6(4.5 \%) \text { Untreated indication } \\
3(3.2 \%)\end{array}$ \\
\hline 10 & Ruiter Ret al, 2012 [10] & $\begin{array}{l}26852(1.3 \%)(\mathrm{Due} \\
\text { to ADR) }\end{array}$ & & ADR \\
\hline 11 & Pedrós C.et al, 2016 [24] & $\begin{array}{l}1976(3.3 \%) \text { (Due to } \\
\text { ADR) }\end{array}$ & $113(5.7 \%)$ & $\begin{array}{l}\text { Acute renal failure }(22.9 \%) \\
\text { Upper GI bleeding }(16.6 \%) \\
\text { Lower GI bleeding }(11.2 \%) \\
\text { Intracranial bleeding }(9.3 \%) \\
\text { Digitalis intoxication }(3.7 \%)\end{array}$ \\
\hline 12 & Ahern F, et al, 2013 [32] & $\begin{array}{l}75(8.8 \%) \text { (due to } \\
\text { ADR) }\end{array}$ & NR & NR \\
\hline 13 & Pedrós C. et al, 2014 [25] & $4.2 \%$ (due to ADR) & $3.2 \%$ & $\begin{array}{l}\text { Type A reactions } 171(91.9 \%) \\
\text { Type B } 15(8.1 \%) \\
\text { Renal \& urinary disorder } 59(29.4 \%) \\
\text { Gastrointestinal disorders } 53(26.4 \%) \\
\text { Nervous system disorders } 21(10.4 \%)\end{array}$ \\
\hline
\end{tabular}




\begin{tabular}{|c|c|c|c|c|}
\hline 14 & $\begin{array}{l}\text { Karuppannan M. et al, } \\
2013 \text { [27] }\end{array}$ & $\begin{array}{l}443(39 \%) \\
(\mathrm{ADR})\end{array}$ & NR & $\begin{array}{l}\text { Treatment Failure } 351(79 \%) \\
\text { ADR } 94(21 \%) \\
\text { Drug overdose } 21(5 \%) \\
\text { Medication error } 15(3 \%)\end{array}$ \\
\hline 15 & $\begin{array}{l}\text { Skoldunger A. et al, } 2015 \\
\text { [34] }\end{array}$ & $536(13 \%)$ & NR & NR \\
\hline 16 & $\begin{array}{l}\text { Marcum Z. et al, } 2012 \\
\text { [33] }\end{array}$ & $\begin{array}{l}68(10 \%) \\
\text { (due to ADR) }\end{array}$ & NR & $\begin{array}{l}\text { Bradycardia }(n=6) \\
\text { hypoglycemia }(n=6) \\
\text { falls }(n=6) \\
\text { mental status changes }(n=6)\end{array}$ \\
\hline
\end{tabular}

Table 3. Drugs and other factors that are associated with drug related hospitalization

\begin{tabular}{|c|c|c|}
\hline Author, year of publication & Drugs that cause DRP & $\begin{array}{l}\text { Factors significantly associated with } \\
\text { admission due to DRPs }\end{array}$ \\
\hline Alghamdy M. et al, 2015 [20] & $\begin{array}{l}\text { Antiepileptic drugs } \\
\text { Paracetamol } \\
\text { Opioid } \\
\text { Benzodiazepine } \\
\text { Antibiotics } \\
\text { Antisecratory drugs } \\
\text { Antipsychotic drugs } \\
\text { Antihypertensive drugs }\end{array}$ & $\mathrm{NR}$ \\
\hline Chan A. et al, 2014 [21] & $\begin{array}{l}\text { Cyclophosphamide }(n=35), \\
\text { Doxorubicin }(n=25) \\
\text { Cisplatin }(n=25) \\
\text { Docetaxel }(n=18) \\
\text { Paclitaxel }(n=16)\end{array}$ & $\begin{array}{l}\text { Young age }(p=0.03) \\
\text { Female sex }(p=0.015)\end{array}$ \\
\hline Schmiedl, S et al, 2014[22] & $\begin{array}{l}\text { Antithrombotic agents } \\
\text { drugs used in diabetes } \\
\text { NSAIDs } \\
\text { Paracetamol }\end{array}$ & NR \\
\hline $\begin{array}{l}\text { Benard-laribiere A. etal, } 2015 \\
\text { [23] }\end{array}$ & $\begin{array}{l}\text { Antithrombotic } 12.6 \% \\
\text { Antineoplastic agents } 12.6 \% \\
\text { Diuretics } 9.0 \% \\
\text { Analgesics } 9.0 \% \\
\text { Anxiolytics/hypnotics/antipsychotics } 6.6 \%\end{array}$ & Old age $(p<0.001)$ \\
\hline ReppK. et al, 2012 [5] & $\begin{array}{l}\text { Immunosuppressant }(63 \%) \\
\text { Antimicroblal }(11 \%) \\
\text { Electrolyte/Fuid }(11 \%) \\
\text { Anticoagulant }(5 \%)\end{array}$ & NR \\
\hline Al-Arifi M. et al, 2014 [4] & $\begin{array}{l}\text { Antihypertensive agents }(21.5 \%) \\
\text { Anticoagulants }(14.3 \%) \\
\text { Immunosuppressant }(12.5 \%) \text { Chemotherapeutic agents } \\
(10.7 \%)\end{array}$ & NR \\
\hline Nickel C. et al, 2013 [26] & $\begin{array}{l}\text { Thiazides } \\
\text { Benzodiazepines } \\
\text { Antidepressants } \\
\text { Anticonvulsants }\end{array}$ & NR \\
\hline Gustafsson M. et al, 2016 [16] & $\begin{array}{l}\text { Cardiovascular drugs (29.5\%) Psychotropic drugs } \\
(27.3 \%)\end{array}$ & Poly-pharmacy \\
\hline Jatau A. et al, 2015 [18] & $\begin{array}{l}\text { Antidiabetics }(23.3 \%) \\
\text { Antihypertensives }(21.1 \%) \\
\text { Antibiotics }(9.8 \%) \\
\text { Anti-asthmatics }(8.3 \%) \\
\text { Diuretics }(6.0 \%)\end{array}$ & NR \\
\hline Ruiter Ret al, 2012 [10] & $\begin{array}{l}\text { Anticoagulants } \\
\text { Antineoplastic and immunosuppressive drugs } \\
\text { Antidiabetic agents } \\
\text { High-ceiling diuretics } \\
\text { Salicylates } \\
\text { Antirheumatics }\end{array}$ & $\begin{array}{l}\text { Age } \geq 75 \text { years } \\
\text { Female sex }\end{array}$ \\
\hline
\end{tabular}


Pedrós C.et al, 2016 [24]

Ahern F, et al, 2013 [32]
Pedrós C. et al, 2014 [25]

Karuppannan M. et al, 2013

[27]

Thiazide diuretic $10(10.6 \%)$
ACE Inhibitor $10(10.6 \%)$
CCB $10(10.6 \%)$

Skoldunger A. et al, 2015 [34] NR
High-ceiling diuretics

ACE inhibitors

Cardiac glycosides

Diuretics $(\mathrm{n}=22)$,

Aspirin ( $\mathrm{n}=5)$

Warfarin $(n=4)$

Diuretics $69(18.1 \%)$

Antithrombotic drugs 63 (16.5\%)

RAAS inhibitors 56 (14.7\%)

NSAIDs 43 (11.3\%)

Antidiabetic $36(38.3 \%)$

Antiplatelet $10(10.6 \%)$

Thiazide diuretic $10(10.6 \%)$

CCB $10(10.6 \%)$
NR

Poly-pharmacy

Age $\geq 65$ years

Poly-pharmacy

NR

Old age,

Male sex,

Living at home (community dwelling),

Lower educational level,

Functional dependence,

Multiple co-morbidity.

$\begin{array}{ll}\text { Marcum Z. et al, } 2012[33] & \text { Beta-blocker } \\ & \text { Oral hypoglycemic agent } \\ & \text { Thiazide diuretic } \\ & \text { Anticoagulant } \\ & \text { Antidepressant } \\ & \text { NSAIDs }\end{array}$

Drugs and Other Factors that Are Associated with Drug Related Hospitalization

Drugs that were frequently reported as causing drug related admission were antithrombotic drugs, antihypertensive drugs, analgesics, anti-diabetics, antipsychotics, and anti-neoplastic drugs. Poly pharmacy, old age and female sex are mentioned as factors for drug related hospitalization by a number of studies. Presence of multiple co morbidity, lower educational level, functional dependence, young age and male sex were also mentioned in some studies as a factor that influence hospital admission due to drug related problems.

\section{Preventability of DRPS that Caused Hospital Admission}

Around one third of drug related hospital admissions were definitely preventable and more than $40 \%$ were potentially preventable. Report of the reviewed studies on the Preventability of Drug related hospitalization is indicated in Table 4.

\section{Discussion}

This systematic review summarizes prevalence of hospital admissions due to drug-related problems and preventability of drug related hospital admissions. In addition, the study has also extorted findings to identify the common types of DRPs that caused hospital admission, and associated factors responsible for drug related hospital admission from 16 different studies published recently.

The rate of drug related hospital admission varies from $1.3 \%$ to $41.3 \%$ with the average rate of $15.4 \%$. This figure indicated that the burden of DRPs as a cause of hospitalization is very significant. In addition to hospital admission, DRP may contribute to poor patients' clinical outcome at discharge such as disability or death. Chiefly ADRs can be potentially lethal and are a main cause of mortality [19]. The mortality rate among patients hospitalized due to drug related problems as reported in the seven studies included in this review [5, 20-25] was up to

Table 4. Preventability of DRPs that caused hospital admission

\begin{tabular}{llll}
\hline Author, year & Not preventable (\%) & Potentially preventable (\%) & Definitely preventable (\%) \\
\hline Alghamdy M. et al, 2015 [20] & NR & 70 & NR \\
Chan A. et al, 2014 [21] & 45.7 & 52.4 & 15.2 \\
\hline Benard-laribiere A. et al, 2015 [23] & 16.5 & 16.5 & 32 \\
ReppK. et al, 2012 [5] & NR & NR & 58 \\
Al-Arifi M. et al, 2014 [4] & 14.3 & 53.6 & 32.1 \\
Jatau A. et al, 2015 [18] & 33.1 & 11.3 & 55.5 \\
Ahern F, et al, 2013 [32] & 33.3 & 52 & 5.3 \\
Marcum Z. et al, 2012 [33] & $\mathrm{NR}$ & $\mathrm{NR}$ & 36.8 \\
Average & $28.6 \%$ & $42.6 \%$ & $33.6 \%$ \\
\hline
\end{tabular}


$5.7 \%$ with the average death rate of $2.7 \%$.

The types of DRPs causing hospital admission varied across the studies reviewed. Among the twelve studies that have reported types of DRPs causing hospital admission, six [4, 5, 16, 20, 21, 26] have explicitly reported the frequencies of each type of DRPs responsible for hospital admission. As a result, ADR in 4 studies $[4,5,21,26]$, noncompliance in Alghamdyet al., [20] study and dosage too high in Gustafssonet al. [16] study were identified as the most frequently occurring types of DRPs that caused hospital admission. However, Jatauet al., [18] and Karuppannanet al., [27] have assessed prevalence of admissions due to ADE and both studies identified treatment failure as the most frequent cause of admission, which is in contrast to previous studies published before 2012 where ADRs was most common type of ADE accounted for 53-90\% of ADE related admissions [1, 28-30] followed by therapeutic failure [31]. The other four studies [10, 23-25] in this review have determined ADR specific hospital admission and hence, Type A reaction in two studies $[23,25]$ and acute renal failure in Pedróset al.,[24] study have been reported to be the most common subtypes of ADR that caused hospital admission.

There have not been any standardized methods of classifying drugs making it difficult for comparisons as to which drug causes drug related hospital admission across different studies. Concordant with the studies included in the present review $[10,16$, $18,24,25,32]$ a previous review that took studies published before 2012 mentions, cardiovascular drugs (antithrombotic drugs, antihypertensive drug), antineoplastic agents, anti-inflammatory and antidiabetic agents as a commonly reported drug classes causing hospital admission [3].

Age is the most frequently mentioned factor for drug related hospital admission. In line with the result of this review old age was identified as a major risk factor for drug related hospital admission in many other studies [3, 7, 12, 13, 17]. The main reason for geriatric population to be more susceptible for admission due to DRPs could be due to physiological and pathological changes occurred at advanced age which leads to a change in pharmacodynamics and pharmacokinetics of a drug in elderly. Drug absorption, distribution metabolism and excretion will be significantly affected in older people which may subsequently lead to toxicity and complication which necessitates hospitalization. In addition polypharmacy [16, 25, 32, 33], gender $[10,21]$, presence of multiple co morbidity [34], lower educational level and functional dependence [34] were also mentioned as a factor that influence hospital admission due to drug related problems. Similarly previous studies also agree that the above mentioned factors have significant association with drug related hospital admission [3, 5, 7, 12-17].

According to the current review significant numbers of DRPs were found to be either potentially or definitely preventable. Hence, several strategies can be designed to minimize those preventable DRPs, thereby curbing the risk of hospital admission. Health care providers should be aware of the common risk factors so as to lower hospital admission due to DRPs. Furthermore, prescribers are in a better position to lessen inappropriate prescribing and identifying the associated factors with DRPs. Pharmacists should also be involved in medication review and reconciliation and actively participate in minimizing poly pharmacy so as to lower the incidence of drug related admissions.

Drug related problems contribute for more than $15 \%$ of hospital admissions. Higher risk of admission due to DRPs was observed in patients who were on poly pharmacy and those who were old. As most of drug related hospital admissions were preventable an emphasis should be given for preventive strategies to avoid costs and complications associated with admission.

\section{Acknowledgment}

We would like to thank University of Gondar for providing us an internet access for searching literatures to realize this review.

\section{Author contributions}

All authors contributed toward literaturesearching, drafting and critically revising the paper and agree to be accountable forall aspects of the work.

Conflicts of Interest: None declared.

\section{References}

1. Bhalla N, Duggan C, Dhillon S. The incidence and nature of drugrelated admissions to hospital. Pharmaceutical Journal. 2003.

2. Roxburgh A, Degenhardt L. Characteristics of drug-related hospital separations in Australia. Drug Alcohol Depend. 2008;92(1-3):149-55.

3. Nivya K, Sri Sai Kiran V, Ragoo N, Jayaprakash B, Sonal Sekhar M.
Systemic review on drug related hospital admissions - A pubmed based search. Saudi Pharm J. 2015;23(1):1-8.

4. Al-Arifi M, Abu-Hashem $\mathrm{H}$, Al-Meziny M, Said R, Aljadhey H. Emergency department visits and admissions due to drug related problems at Riyadh military hospital (RMH), Saudi Arabia. Saudi Pharm J. 2014;22(1):17-25.

5. Repp KL, Hayes C 3rd, Woods TM,
Allen KB, Kennedy K, Borkon MA. Drug-related problems and hospital admissions in cardiac transplant recipients. Ann Pharmacother. 2012;46(10):1299-307.

6. Conforti A, Costantini D, Zanetti F, Moretti U, Grezzana M, Leone R. Adverse drug reactions in older patients: an Italian observational prospective hospital study. Drug Healthc Patient Saf. 2012;4:75-80. 
7. Davies EC, Green CF, Mottram DR, Rowe PH, Pirmohamed M Emergency re-admissions to hospital due to adverse drug reactions within 1 year of the index admission. $B r J$ Clin Pharmacol. 2010;70(5):749-55.

8. Pérez Menéndez-Conde C, Bermejo Vicedo T, Delgado Silveira E, Carretero Accame E. Adverse drug reactions which provoke hospital admission. Farm Hosp. 2011;35(5):236-43.

9. osthumus AA, Alingh CC, Zwaan CC, van Grootheest KK, Hanff LL, Witjes $\mathrm{BB}$, et al. Adverse drug reactionrelated admissions in paediatrics, a prospective single-centre study. $B M J$ Open. 2012;2(4). pii: e000934.

10. Ruiter R, Visser LE, Rodenburg EM, Trifiró G, Ziere G, Stricker BH. Adverse drug reaction-related hospitalizations in persons aged 55 years and over: a population-based study in the Netherlands. Drugs Aging. 2012;29(3):225-232.

11. Stausberg J, Hasford J. Drug-related admissions and hospital-acquired adverse drug events in Germany: a longitudinal analysis from 2003 to 2007 of ICD-10-coded routine data. BMC Health Serv Res. 2011;11:134.

12. Hartholt $\mathrm{KA}$, van der Velde $\mathrm{N}$, Looman CW, Panneman MJ, van Beeck EF, Patka P, et al. Adverse drug reactions related hospital admissions in persons aged 60 years and over, The Netherlands, 1981-2007: less rapid increase, different drugs. PLoS One. 2010;5(11):e13977.

13. Carrasco-Garrido $P$, de Andrés LA, Barrera VH, de Miguel GA, JiménezGarcía R. Trends of adverse drug reactions related-hospitalizations in Spain (2001-2006). BMC Health Serv Res. 2010;10:287.

14. Wu C, Bell CM, Wodchis WP. Incidence and economic burden of adverse drug reactions among elderly patients in Ontario emergency departments: a retrospective study. Drug Saf. 2012;35(9):769-81.

15. Rodenburg EM, Stricker BH, Visser LE. Sex-related differences in hospital admissions attributed to adverse drug reactions in the Netherlands. Br J Clin Pharmacol. 2011;71(1):95-104.

16. Gustafsson M, Sjölander M, Pfister B, Jonsson J, Schneede J, Lövheim
H. Drug-related hospital admissions among old people with dementia. Eur J Clin Pharmacol. 2016;72(9):1143-53.

17. Wu TY, Jen MH, Bottle A, Molokhia M, Aylin P, Bell D, et al. Ten-year trends in hospital admissions for adverse drug reactions in England 1999-2009. J R Soc Med. 2010;103(6):239-50.

18. Jatau AI, Aung MM, Kamauzaman TH, Rahman AF. Prevalence of DrugRelated Emergency Department Visits at a Teaching Hospital in Malaysia. Drugs Real World Outcomes. 2015;2(4):387-395.

19. Lazarou J, Pomeranz BH, Corey PN. Incidence of adverse drug reactions in hospitalized patients: a metaanalysis of prospective studies. JAMA. 1998;279(15):1200-5.

20. Alghamdy MS, Randhawa MA, Al-Wahhas MH, Al-Jumaan MA. Admissions for drug-related problems at the Emergency Department of a University Hospital in the Kingdom of Saudi Arabia. J Family Community Med. 2015;22(1):44-8.

21. Chan A, Soh D, Ko Y, Huang YC, Chiang J. Characteristics of unplanned hospital admissions due to drug-related problems in cancer patients. Support Care Cancer. 2014;22(7):1875-81.

22. Schmiedl S, Rottenkolber $M$, Hasford J, Rottenkolber D, Farker K, Drewelow B, et al. Self-medication with over-the-counter and prescribed drugs causing adverse-drug-reactionrelated hospital admissions: results of a prospective, long-term multi-centre study. Drug Saf. 2014;37(4):225-35.

23. Bénard-Laribière $A$, MiremontSalamé G, Pérault-Pochat MC, Noize P, Haramburu F; EMIR Study Group on behalf of the French network of pharmacovigilance centres. Incidence of hospital admissions due to adverse drug reactions in France: the EMIR study. Fundam Clin Pharmacol. 2015;29(1):106-11.

24. Pedrós C, Formiga F, Corbella X, Arnau JM. Adverse drug reactions leading to urgent hospital admission in an elderly population: prevalence and main features. Eur J Clin Pharmacol. 2016;72(2):219-26.

25. Pedrós $C$, Quintana $B$, Rebolledo M, Porta N, Vallano A, Arnau JM.
Prevalence, risk factors and main features of adverse drug reactions leading to hospital admission. Eur J Clin Pharmacol. 2014;70(3):361-7.

26. Nickel CH, Ruedinger JM, Messmer AS, Maile S, Peng A, Bodmer M, et al. Drug-related emergency department visits by elderly patients presenting with non-specific complaints. Scand $J$ Trauma Resusc Emerg Med. 2013;21:15.

27. Karuppannan M, Nee TK, Ali SM, Thong WK, Boardman H. The prevalence of adverse drug eventrelated admissions at a local hospital in Malaysia. Archives of Pharmacy Practice. 2013;4(4):160.

28. Saha L, Pandhi P, Malhotra S, Sharma N. Adverse Drug Event (ADE) related Medical Emergency Department visits and hospital admissions: a prospective study from a North Indian Referral Hospital. J Clin Diag Res. 2008;2(2):600-4.

29. Chan M, Nicklason F, Vial JH. Adverse drug events as a cause of hospital admission in the elderly. Intern Med J. 2001;31(4):199-205.

30. Hallas J, Gram LF, Grodum E, Damsbo N, Brøsen K, Haghfelt T, et al. Drug related admissions to medical wards: a population based survey. $\mathrm{Br}$ J Clin Pharmacol. 1992;33(1):61-8.

31. Zargarzadeh AH, Emami $\mathrm{MH}$, Hosseini F. Drug-related hospital admissions in a generic pharmaceutical system. Clin Exp Pharmacol Physiol. 2007;34(5-6):494-8.

32. Ahern F, Sahm LJ, Lynch D, McCarthy S. Determining the frequency and preventability of adverse drug reaction-related admissions to an Irish University Hospital: a cross-sectional study. Emerg Med J. 2014;31(1):24-9.

33. Marcum ZA, Amuan ME, Hanlon JT, Aspinall SL, Handler SM, Ruby $\mathrm{CM}$, et al. Prevalence of unplanned hospitalizations caused by adverse drug reactions in older veterans. $\mathrm{J} \mathrm{Am}$ Geriatr Soc. 2012;60(1):34-41.

34. Sköldunger A, Fastbom J, Wimo A, Fratiglioni L, Johnell K. Impact of Inappropriate Drug Use on Hospitalizations, Mortality, and Costs in Older Persons and Persons with Dementia: Findings from the SNAC Study. Drugs Aging. 2015;32(8):671-8. and share. Bulletin of Emergency And Trauma articles are published under a Creative Commons license (CC-BY-NC). 